Wettbewerbsfähigkeit von Unternehmen im europäischen Markt

\title{
Marktverzerrungen durch Umweltpolitik?
}

\author{
Unternehmen beklagen sich häufig über Wettbewerbs- \\ verzerrungen durch Umweltpolitik. Tatsächlich hat \\ Umweltpolitik jedoch das Potenzial einheitliche Rahmen- \\ bedingungen auf dem europäischen Markt zu schaffen und \\ Wettbewerbsverzerrungen zu beseitigen. \\ Von Klaus Jacob und Jesko Eisgruber
}

D ie neue Europäische Kommission hat eine umfassende umweltpolitische Agenda. Dazu zählt unter anderem die Revision wichtiger Richtlinien oder klimapolitischer Maßnahmen. Allzu häufig beklagen Unternehmen Wettbewerbsverzerrungen, die durch Umweltpolitik auftreten. Inwieweit sind diese Klagen bei den aktuellen Regulierungsvorhaben berechtigt? Werden durch europäische Umweltpolitik die Wettbewerbsbedingungen von Unternehmen beeinflusst und wie verhält sich dies zu den Zielen eines einheitlichen Europäischen Marktes?

Inwieweit ist eine weitergehende Harmonisierung europäischer Umweltpolitik anzustreben und kann und soll dabei auch auf die Vertragsgrundlagen zurückgegriffen werden, die den einheitlichen Europäischen Markt sicherstellen?

In der Europäischen Union (EU) wird Umweltpolitik zwar von den europäischen Institutionen formuliert und beschlossen, die Umsetzung erfolgt aber im Regelfall auf der nationalen Ebene. Den Mitgliedsstaaten wird dabei die Freiheit eingeräumt, innerhalb der durch europäisches Recht vorgegebenen Grenzen die abgeleiteten Normen anzupassen. Diese Flexibilität bei der Umsetzung hat den Vorteil, dass die Zustimmungsfähigkeit für Umweltnormen in den europäischen Institutionen steigt. Allerdings besteht die Möglichkeit, dass die Umsetzung von EU-Richtlinien zwischen den Mitgliedsstaaten divergiert.

Die Europäische Kommission, Generaldirektion Umwelt, beauftragte eine Stu- die zum Verhältnis von Umweltpolitik und dem einheitlichen europäischen Markt. Dabei galt es zunächst, eine Definition für Marktverzerrungen zu erarbeiten. Als Marktverzerrung wird gelegentlich jede Veränderung der relativen Wettbewerbsfähigkeit bezeichnet. Besonders Unternehmen und ihre Verbände, welche von neuen oder verschärften Standards betroffen sind, beklagen sich aus dieser Perspektive über Umweltnormen.

\section{Relativer Wettbewerb und Marktverzerrung}

In einem engeren und auch hier verwendeten Sinne werden unter Marktverzerrungen allerdings nur Situationen verstanden, bei denen Unternehmen unter ungleichen Bedingungen operieren müssen und diese nicht durch das eigene Handeln, also durch Innovationen oder Investitionen, verändern können. Die Ursachen hierfür können vielfältig sein, beispielsweise Monopole, Handelsbarrieren, Regulationen oder Subventionen.

Wir unterscheiden vier idealtypische Situationen, in denen Umweltnormen die relative Wettbewerbsfähigkeit von Unternehmen beeinflussen. Nur zwei dieser Situationen können unserem Verständnis nach als Marktverzerrung bezeichnet werden.

I Der erste Fall beschreibt die Möglichkeit, dass durch Fehlen von einheitlicher europäischer Umweltpolitik in einem Land die Kosten für die Nutzung von natürlichen Ressourcen geringer sind als dort, wo aufgrund von umweltpolitischen Normen die Kosten der Ressourcennutzung internalisiert werden. Dies stellt eine Wettbewerbsverzerrung dar. Eine Vereinheitlichung umweltpolitischer Maßnahmen durch europäische Politik verändert zwar die relative Wettbewerbsfähigkeit, beseitigt aber die Marktverzerrungen. Dies kann zunächst zu einem Wettbewerbsnachteil für jene Unternehmen führen, die sich auf fehlende oder schwächere Umweltstandards eingestellt haben und sich jetzt dem Wettbewerb mit Unternehmen ausgesetzt sehen, die schon seit ehedem anspruchsvollere Umweltnormen einhalten müssen. Allerdings können sich Unternehmen durch Innovationen an diese neue Situation anpassen. Die deutsche Automobil-Industrie sieht sich beispielsweise durch den einheitlichen europäischen Emissionsstandard von 120 Gramm pro Kilometer unverhältnismäßig benachteiligt. Eine Marktverzerrung stellt dies jedoch nicht dar, da sich die deutsche Industrie, wie auch Wettbewerber in Frankreich oder Italien, auf die Herstellung von kleineren und sparsameren Autos einstellen kann.

I Im zweiten Fall wird europäische Umweltpolitik nicht oder nicht in dem vorgesehenen Maße national umgesetzt. In der Konsequenz entstehen dadurch Nachteile für Unternehmen, die in Ländern produzieren, die Umweltnormen vollständig übernommen haben. Dieser Tatbestand stellt eine Marktverzerrung dar, die nicht durch unternehmerisches Handeln ausgeglichen werden kann. Einen solchen Fall konnten wir bei der Analyse der nationalen Umsetzung des Europäischen Emissionshandels für den Fall der Zementindustrie beobachten. Die Überallokation von Emissionszertifikaten führt zu Wettbewerbsvorteilen für die Zementindustrie in Ländern mit wenig anspruchsvollen Reduktionsplänen. Dies stellt eine Marktverzerrung dar.

- Ein dritter Fall beschreibt die Situation, in der eine Umweltnorm in einem Mitgliedsstaat so eingeführt wird, 
dass sie größeren bürokratischen Aufwand für heimische Unternehmen verursacht als dies in anderen Mitgliedsstaaten der Fall ist. So kann beispielsweise die Höhe von Gebühren oder Informationspflichten zwischen den Mitgliedsstaaten divergieren. Auch hier wird ein dauerhafter Wettbewerbsnachteil geschaffen, der durch unternehmerisches Handeln nicht auszugleichen ist. Ein Beispiel für diesen Fall von Wettbewerbsverzerrung ist die Richtlinie zur Rücknahme von Elektroabfällen WEEE. Die Rücknahmesysteme unterscheiden sich deutlich voneinander und entsprechend die damit verbundenen Kosten für Unternehmen.

- Schließlich untersuchten wir noch einen vierten Fall der Beeinflussung der Wettbewerbsfähigkeit durch Umweltnormen. Wenn ein Land über den auf europäischer Ebene vereinbarten Standard hinausgeht oder zusätzliche Maßnahmen ergreift, werden dadurch höhere Kosten für heimische Unternehmen verursacht. Wir argumentieren jedoch, dass dies nicht als eine Marktverzerrung gewertet werden kann, da die Norm freiwillig überschritten wird.

Die Beurteilung der Frage, ob eine Marktverzerrung vorliegt oder nicht, lässt sich nicht alleine aus harten Indikatoren ablesen, etwa den Preisen für natürliche Ressourcen. Diese können sich auch aufgrund von natürlichen oder strukturellen Bedingungen unterscheiden und nicht alleine aufgrund von Unterschieden zwischen den umweltpolitischen Standards. Eine Marktverzerrung in unserem Sinne liegt nur dann vor, wenn dem eine unfaire Unterschreitung der kollektiv vereinbarten Norm zugrunde liegt, nicht jedoch wenn die Überschreitung der Norm aufgrund der Präferenzen eines Landes erfolgt.

\section{Wie Umweltpolitik Marktver- zerrungen beseitigt}

Die Frage, ob Marktverzerrungen vorliegen oder nicht, lässt sich in vielen Fällen nicht oder zumindest nicht ex-ante be- antworten. Eine notwendige Voraussetzung der Bewertung ist, dass es einen europäischen Standard gibt. Wenn in den Europäischen Richtlinien nur ein Prozess oder ein bestimmter Umweltzustand definiert wird, ohne Instrumente vorzugeben, ist eine Abschätzung von Marktverzerrungen durch eine Unterschreitung der europäischen Ziele ex ante nicht möglich. Aus der Sicht des einheitlichen europäischen Marktes ist es wünschenswert, wenn in Umweltdirektiven klare Ziele vereinbart werden, selbst wenn das Instrumentarium zu deren Erreichung den Mitgliedsstaaten freigestellt bleibt.

Mehrere unserer Fallstudien zeigen, dass die Europäisierung von Umweltpolitik, trotz der unterschiedlichen nationalen Umsetzung, ursprünglich bestehende Marktverzerrungen zu beseitigen half und daher einen Anteil an der Schaffung des gemeinsamen europäischen Marktes hatte. Europäische Umweltpolitik hat somit das Potenzial einheitliche Rahmenbedingungen auf dem europäischen Binnenmarkt zu schaffen, Marktverzerrungen zu beseitigen oder zu verhindern.

\section{Anmerkung}

Dieser Artikel bezieht sich auf die Ergebnisse der Studie:

Jacob, K. et al.: Environment and the Single Market. Final Report to the European Commission by Freie Universität Berlin, Adelphi Research, University of Konstanz, GHK and PSI. Berlin, Konstanz and London 2009.

\section{AUTOREN + KONTAKT}

Dr. Klaus Jacob ist Forschungsleiter und Jesko Eisgruber ist Wissenschaftlicher Mitarbeiter an der Forschungsstelle für Umweltpolitik der Freien Universität Berlin.

Forschungsstelle für Umweltpolitik (ffu), Innestraße 22, 14195 Berlin. Tel.: +49 3083854492

E-Mail: klaus.jacob@fu-berlin.de, E-Mail: jesko.eisgruber@fu-berlin.de, Internet: http://www.fu-berlin.de/ffu

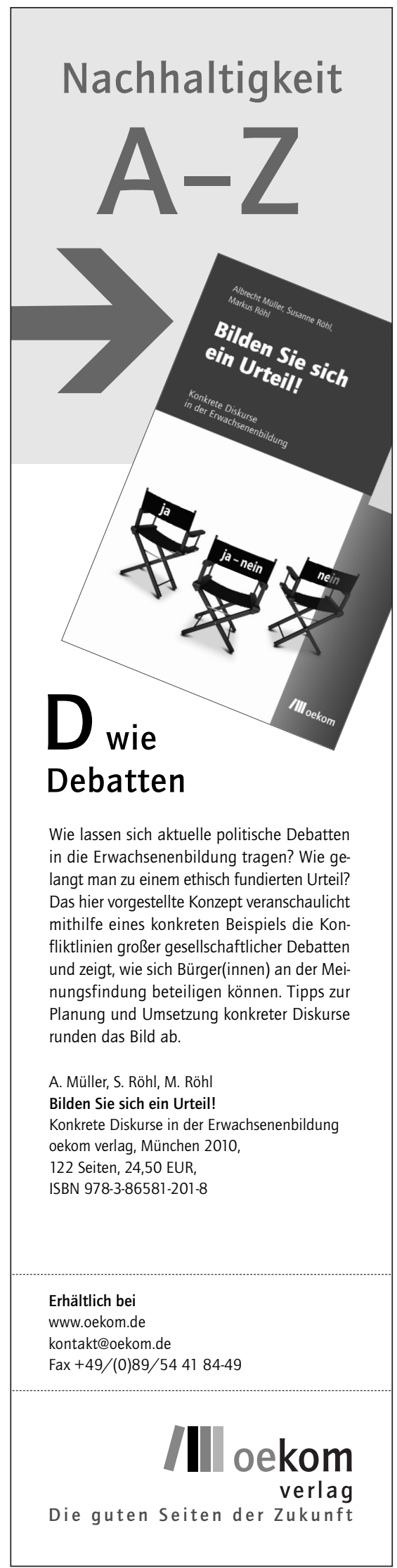


(c) 20I0 Authors; licensee IÖW and oekom verlag. This is an article distributed under the terms of the Creative Commons Attribution Non-Commercial No Derivates License (http://creativecommons.org/licenses/by-nc-nd/3.o/), which permits unrestricted use, distribution, and reproduction in any medium, provided the original work is properly cited. 